\title{
A COMPREHENSIVE REVIEW OF ENERGY-BASED ROUTING STRATEGIES FOR INTERNET OF THINGS
}

\author{
Srinivasulu $\mathrm{M}^{1}$, Dr. Shivamurthy $\mathrm{G}^{2}$ \\ ${ }^{1}$ Department of MCA, University BDT College of Engineering, Davanagere, Karnataka, India. \\ ${ }^{2}$ Department of MCA, VTU PG Center, Muddenahalli, Chickkballapur District, Karnataka, India
}

\begin{abstract}
IoT is the acronym for Internet of Things acronym. At present IoT is a buzzword amongst academia, research and industry communities. Everything surrounded by us have developed abilities to communicate via the medium of internet. The Routing information plays a vital role in establishing communication between nodes in the space of IoT. Maximum energy of such connected nodes is consumed in the process of routing the packets. In this context optimizing the network lifetime with minimal energy consumption becomes important for efficient implementation of IoT infrastructure. This literature review is has the objective to identify the limitations existing in improving the network usability and thus enhance the network lifetime. The focus of this review is to consider various parameters like Quality of Service (QoS), efficient node deployment techniques, Network lifetime for Wireless Sensor Networks (WSN). A comprehensive and systematic study of Routing challenges encountered in an IoT network is accomplished. Further the performance of various energy routing protocols are studied.
\end{abstract}

Keywords: Internet of Things (IoT), Standards, IoT Routing Protocols, Wireless Sensor Networks (WSN), Energy Efficiency, Network Lifetime.

\section{Introduction}

Internet of Things (IoT) has synonymous to the happening trend in technology frontier. IoT has made human race to realize many of its dreams of having ready-to-use smart devices that can connect any of the devices in our surroundings to Internet. Also IoT has helped humanity to deploy such interconnected nodes in any environment with communication and collaborating capabilities. Such development in technology arena could be realized due to the current developments in the fields of sensing devices, machine to machine communication, internet protocols, and big data analytics. As stated in the 2015 Gartner report, the IoT domain has enabled such vision and proved lucrative by as billions of dollars is being invested in this domain with high research perspective [35]. The term IoT in nutshell is coined using two words: "internet" and "things". Meaning it facilitates things, or non-computing devices, to develop sensory features like hear, see, think and also compute. They can establish communication and coordination channels with each other in decision making. Finally such inanimate things can also act as smart devices and involve in taking consensus decisions beneficial for different applications. IoT can convert objects or sensors from being passive players into active devices that can compute, communicate, collaborate and take vital decisions. The elementary technologies behind the success of IoT are powerful embedded sensors, evolution of novel computing models, analytics, less intensive communication, and innovative development in internet protocol standards. However, the challenge emerged from these developments direct the researchers to show interest on developing dedicated standards and communication protocols to contrive the new challenges.

In recent developments in the arena of IoT, researchers are focusing on developing applications that suit the market demands and customer requirements. Importantly, the nodes used should be mature enough to adjust for the user demand for round the clock availability and accessibility from anywhere. Also developing new techniques are vital for establishing compatible communication between heterogeneous nodes [28]. The existing methods suggest different solutions to alleviate the current IoT challenges. Important solutions developed address data aggregation, data transmitting methods, clustering, and applying cross-layer designing and automating things. Predominantly the existing solutions use dynamic clustering methods for data collection in the wide area WSNs. Clusters are formed by aggregating sensor nodes. Any cluster comprises of Cluster Head as a pioneer and other nodes as members. In addition to balance the network load on cluster head topology is frequently rearranged [23].

IoT has a wide array of roles today's world. Applications including smart home, Smart Manufacturing, Energy Management, Smart City, Smart Environment, Smart Agriculture, Medical \& Health-Care and Transportation as shown in the figure 1 are few of the important IoT applications that can change our lifestyles and also improve quality of life. Best available example in the current IoT ecosystem is smart homes. Smart Homes operates using sensors and controls temperature and other appliances like fans, TV etc in our homes remotely. In future we can envision Smart Homes where beverage preparation, tracking health statistics can be achieved remotely through IoT. Even driverless cars are part of IoT ecosystem. Thus emerges the urgent need for developing standards that can handle the diversified applications.

The salient feature of IoT network is its ubiquitous nature. IoT networks enable connectivity between smart- 
devices and items with the cloud. Wireless Sensor Networks is a mechanism that collects and communicates data for monitoring and controlling the objective world to ensure better lifestyles [20]. The population explosion in urban areas creates a huge demand for modern infrastructure with appropriate services to ensure congestion free life for citizens. This is as pushed the Governments to utilize advancements in communication technologies such as IoT to develop a framework for to build smart cities [34]. As depicted in the figure 1 various IoT applications promise can enhance quality of life. The developments of novel wireless networks, superior ability sensors and path breaking computing capabilities, The IoT can be the next cutting edge technology offering lucrative careers.

- Smart Home: Most popular applications of IoT are Smart Homes. With smart home concept every appliance of our house is connected to the internet \& can be remotely governed. In nutshell a smart home is a 'technology-driven home' where almost all home appliances are controlledcoordinated automatically and remotely by connecting to internet. Also IoT and wireless sensor network combo has the ability to provide intelligent energy management solutions for entire buildings. Building administration is possible through laptop or smart phones by accessing energy information and controlling all systems. [39].

- Smart Agriculture: Several studies on the development of new technologies for agriculture application have been carried out recently. It is already known that agriculture is the sector that influences in a very significant way the development of Brazil, but it's also the sector that consumes the most water in the country [17]. IoT has wide spectrum of applications in agriculture namely soil moisture monitoring, artificially controlling climatic conditions to maximize yield, humidity and temperature levels control systems for prevention of fungus. IoT systems can monitor weather conditions and is useful for predicting rain, snow fall, and drought situation. A typical example of IoT application in Agriculture domain is smart cattle farm which monitors cattle movement, behavior, fertility, and lactation and other characteristics and transfers the information to the stakeholders through smart phones thus enhancing the productivity of cattle.

- Smart City: Implementation of Smart cities has become dream-project for Governments of both developed and developing countries. The concept involves widespread use of intensive computational and communicating resources for enriching the life style of the people across the globe [16]. The smart city is a comprehensive and an inclusive concept of smart homes, smart traffic management, intelligent-disaster management, smart utilities, and smart street light, virtually operational power plants in smart grids, smarter emergency systems and smarter health services. Smart city implementation requires good planning throughout the project implementation cycle with proper coordination between all the agencies involved.

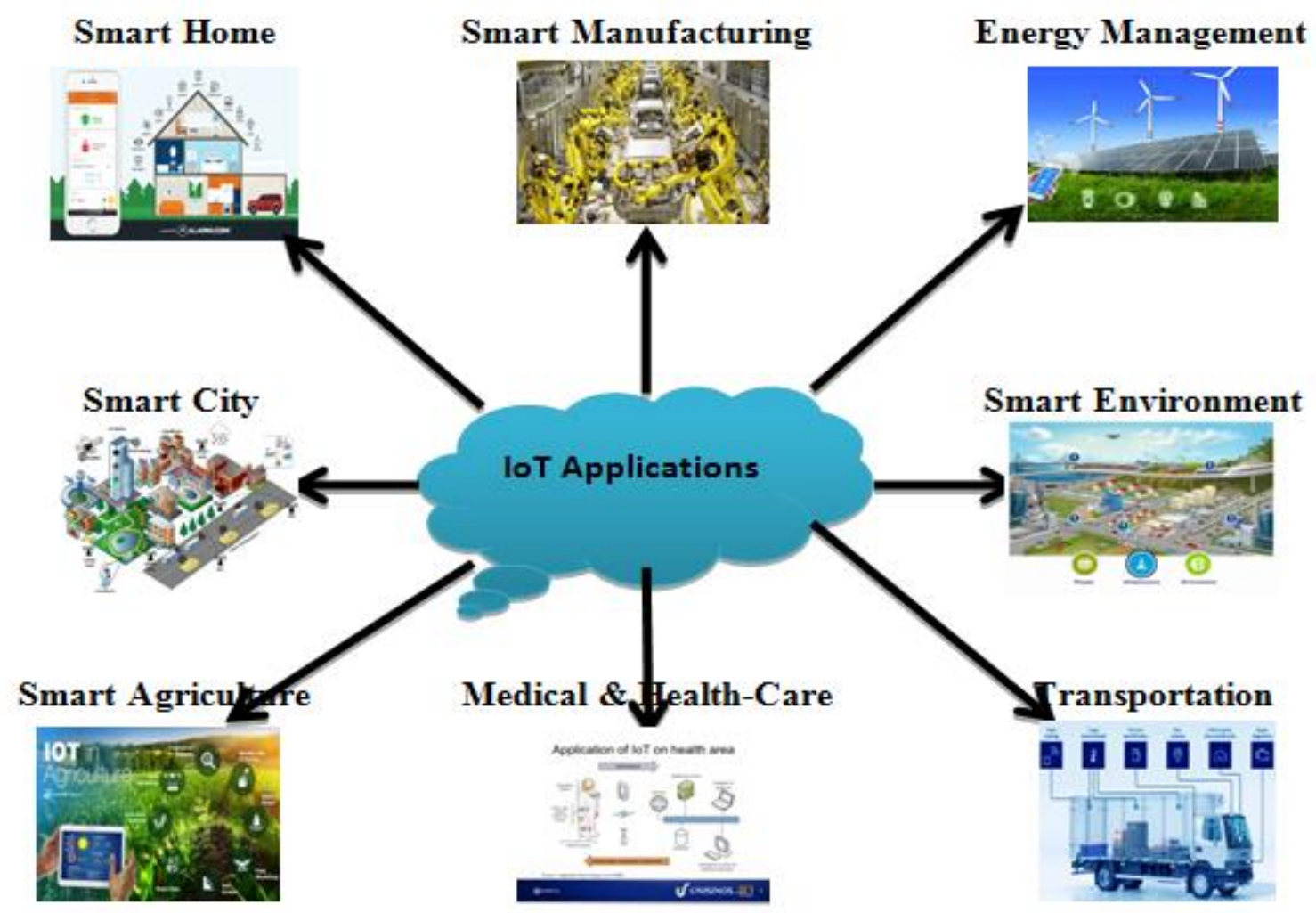

Fig. 1 Applications of Internet of Things 
Smart Manufacturing: Industry operates without any hassles if the manufacturing process and supply chain system are operational effectively. In this context Smart

- $\quad$ factory and Smart Supply chain together constitute Smart Manufacturing system. Smart Manufacturing offers an open platform for both smart factories and supply chains. It also

- facilitates customers and all the stakeholders through rapid and widespread information dissemination across the system. Obviously such system can be envisioned only through usage of the cutting-edge technologies in the entire life cycle [8]. Smart manufacturing system can be realized through a combination of various domains like data analytics, robotics, machine learning, deep learning, sensor technologies, and IoT. Employing these technologies in a cooperative manner helps optimization of manufacturing processes, supports the manufacturers, and assures employee safety.

- $\quad$ Energy Management: Energy is invaluable resource today and Energy management has gained wide acceptance as a research area. Home energy management systems are used to optimize the energy consumption by controlling various appliances like lights, geysers stoves etc. at homes using IT technology. Smart-lighting switches are a best example for such Energy Management system [18]. Energy sector has prominence from all stakeholders namely consumers, businesses and governments. In addition to other benefits offered by the electric power supply chain IoT energy management systems also facilitate development of Smart Grids. The Smart-Grids offers various advantages like power savings, enhanced security and improved efficiency.

- Smart Environment: Conserving Forest and saving environment has gained paramount importance across the globe. Despite various steps initiated by agencies and governments forest fires are rampant. Fire monitoring with the help of WSN, functions by transmitting environmental parameters like barometric pressure, temperature, and relative humidity to the base stations [6]. Importantly Beat Sensors used in smart monitoring systems can identify air pollution gases. Such sensors are capable to monitor the concentrations of different kinds of air pollution gases like $\mathrm{CO}, \mathrm{SO} 2, \mathrm{NOX}$ etc. [7]. Also natural disasters like earthquakes, landslides, can be detected and prevented with these sensors. IoT based systems are also useful to monitor environmental conditions. Industry pollution and vehicular pollution through $\mathrm{CO} 2$ emissions can also be monitored and kept in check by using IoT enabled systems.

- $\quad$ Medical \& Health-Care: Today Health Care and Medical fields are relying on technology advancements to deliver efficient health service to the patients. Various medical services can be coordinated and controlled by intelligently utilizing the services of IoT. For instance medical information can be exchanged between different locations and by diagnosing the diseases proper medications can be administered to patients residing at remotest rural places also. IoT systems facilitate providing constant attention to hospitalized patients whose physiological status needs continuous monitoring. The smart health sensors exhibit the capability to gather comprehensive physiological information and utilize the gateways and the cloud for analyzing and storing the information. Further the analyzed data is sent in wireless mode to the doctors and staff for further analysis.
This system can completely replace the need for a regular health professional's visits at customary intervals to monitor the patient's crucial signs. Thus ensuring automatic information dissemination to all the stakeholders. The system can be beneficial in two ways one the quality of care is improvised through continuous attention and two minimizes the cost of health care with provision for data collection and analytics [19].

- Transportation: Public Transport and Traffic Management is a hurricane task in metropolitan cities. The technology enablement through public IoT networks can bring phenomenal changes to the traffic conditions. Also weather updates help the commuters and drivers to plan their travel. Such IoT system can also collect feedback from the passengers on the driver's behavior and driving style. IoT sensors integrated in each public transport also can record and collect the current bus/cab conditions. Such information helps in providing a cost effective and eco-friendly public transportation network [9]. Also IoT finds various applications like finding the items in big warehouses, verifying the quality of goods shipped, monitoring transportation routes for precious and delicate goods like gold, medicines etc.

\section{History of IoT:}

The IoT domain has opened up plethora of opportunities in technology and communication arena. Through IoT, objects can communicate, compute and transform the information as per the arising needs. The term Internet of Things was first used by Kevin Auston, the Executive Director of Auto-ID Labs at Massachusetts Institute of Technology in 1999. But this concept gained popularity from the Auto-ID centre in 2003 and through their related market analytics and publications [27]. Once the concept of such communication was realized different companies visualized its potential and started investing on this technology [40].

\section{Internet of Things: Protocols and Standards}

a) IEEE 802.15.4e: A data-link layer standard employed at MAC layer. This standard provides the headers, frame format, source and destination addresses. Also it recognizes the communication process occurring between the nodes. Since the conventional frame formats employed in regular computer networks are unsuitable for power constrained IoT devices a separate format in form of IEEE 802.15.4e was shaped as an extension to IEEE 802.15.4. Importantly it supports low power communication. Moreover time harmonization and channel hopping are supported for ensuring high reliability and minimal cost communication in data links [36].

b) IEEE 802.11ah: IEEE 802.11ah is the slightest overhead version of IEEE 802.11 standards. The lightweight nature makes it suitable for IoT applications. IEEE 802.11 standards are popularly termed as Wi-Fi. They are most popular universal wireless standards in conventional computer networks. They are widely employed for almost all digital devices like mobiles, palmtops, digital Televisions, and laptops. But in its original format Wi-Fi standards are unsuitable for IoT applications as they have frame overhead and consume more power. So, 802.11ah mission group was initiated to design a standard supporting low overhead and facilitates energy friendly communication for sensors and nodes [29]. 
c) Wireless HART: Wireless HART standard operates at MAC layer and is layered upon IEEE 802.15.4 PHY. It employs the Time Division Multiple Access (TDMA) method. Also advanced encryption algorithms are put into work for encrypting the messages and for integrity verification. Obviously, Wireless HART possess more secure and reliable features than its counterparts. Fig. 3 depicts the architecture of Wireless HART. Basically it comprise of a security manager, network manager, a gateway, routers, adapters, field devices and access points. This standard provides end-to-end, per-hop or peer-to-peer security features. While the point to point security feature enforces source to destination security the perhop feature provides security to the next designated hop [42], [30].

d) Bluetooth Low Energy: This standard is employed for data link layer. Basically it is a short-range communication standard used widely in IoT or Bluetooth smart. Predominantly they are used for in-vehicle networking. Moreover, the latency is 15 times smaller than the classic Bluetooth standards. The access control of this standard operates in contention-less MAC and exhibits low delay and speed transmission. A master or slave architecture of this standard provides two types of frames: 1) adverting and 2) data frames. The advertise frame carries out discovery slave nodes communicate this signal various devoted advertisement channels. The Master nodes sense the signal from advertising channels and find slaves and establish connection with them. On successful connection establishment the master signals the slave about waking cycle and scheduling sequence. Always the nodes are awake during communication mode and otherwise they remain in sleep mode to save the power [41], [37].

e) ZigBee Smart Energy: It is one of the accepted standard used in IoT systems. It has a dedicated medium-range communication for smart homes, gadget remotes, and healthcare related arrangements. The various networking topology employed by ZigBee are star, peer-to-peer, and cluster-tree. In this standard a centrally located coordinator controls the network for a star topology. The root node controls the network in tree topology or cluster topology. The peer-to-peer topology utilizes the services of coordinator that can be located anywhere. The ZigBee standard possesses two types of stack profiles: ZigBee and ZigBee Pro. The functionality of stack profiles is full mesh network supports and gels well with different applications. The implementation ensures low memory and processing power consumption. ZigBee Pro comes with wide set of security features. Important ones are symmetric-key exchange, scalability through stochastic addresses [24].

f) LoRaWAN: LoRaWAN is a novel wireless technology for long-range wide-area networks and is designed with the objectives of implementing power conserving, minimal cost, mobility, security, and bidirectional communicating IoT networks. It is designed for scalable wireless networks and moreover it is a low-power consumption optimized protocol functional on millions of devices. Various technologies supported by LoRaWAN are energy harvesting, low power consuming,, low cost, location free and redundantly operating ones so that potential needs of IoT for permit mobility and hassle free operations is realized [31]. g) RPL: Routing protocol for low-power and lossy networks (RPL) are a routing protocol that functions with distancevector technique. It is basically designed at IETF to handle route procedure in IoT systems. This protocol is compatible with the entire MAC layer protocols listed above and even some other protocols designed for IoT systems. The working principle of this protocol is based on destination-oriented directed-acyclic graphs in short DODAG. Such graphs possess a single route from every leaf node to the root to carry all the traffic coming from the leaf nodes. Here, every node sends a DODAG information object or DIO to advertise itself as the root. The DODAG network is built in a gradual phase and this is realized by DIO propagation on the network. During communication, DAO - Destination Advertisement Object is transferred to the parents from the node and further propagated to the root. The root takes the decision of routing based on the target. Any nodes wishing to connect to the network will send a DODAG information solicitation (DIS) request and the root acknowledges through a DAO acknowledgment (DAO-ACK) confirmation. Further RPL nodes commonly exhibit stateless nature. Moreover a stateless node can track the status of only parents. In this communication the root alone has the complete knowledge of DODAG. Here the entire communications passes through the root. Also, common nodes can track information about children and parents. Thus while communicating within a sub-tree of the DODAG they need not have to pass through the root [38].

i) IPv6 over Bluetooth Low Energy: RFC 7668 [32] format and IPv6 format are identical. Moreover majority of the 6LowPAN compression techniques are reused. In the Bluetooth Fragmentation occurs in the logical link control and adaptation protocol (L2CAP) sub layer. So, the 6LowPAN fragmentation feature is not employed. In addition, the current version of Bluetooth low energy standards does not assist multi-hop networks formation at the link layer. Rather, a central node is used as a router amongst the peripheral nodes operating with lower-power. In nutshell, the multi-hop feature of 6LowPAN is not applicable here.

\section{Features and characteristics of the IoT:}

The IoT Features and characteristics are documented here [22]:

- Interconnectivity: The key feature of IoT being interconnection of anything to the globally accessible information and communication networks is feasible.

- Things-related services: The IoT operates within the limitations of things namely privacy-protection and semanticconsistency. Through this it provides things-related services by for physical and their associated virtual things. Moreover the technological features of both physical and information world will be changed.

- Heterogeneity: The heterogeneity is the important characteristic of IoT devices. This is obvious as IoT system is built using different hardware platforms and networks. Also they communicate with further services and components using various networks.

- Dynamic changes: Dynamicity is another important characteristic of IoT systems. In IoT networks the device states change dynamically. For instance nodes move quickly to sleep mode and wake up modes or connected and/or disconnected states. Even with additional location and 
changing speed. Importantly, the amount of devices also keeps changing with dynamism.

- Enormous scale: The Magnitude of network is another important characteristic of IoT systems. Here the count of devices to be managed and communicated with other nodes will be of enormous magnitude almost of the size of networks connected to the current Internet. Most importantly managing the data generated and interpreting them is a critical step from the applications point of view. Thus, data semantics efficient data handling also play vital role here.

- Safety: Safety is the next vital characteristic of IoT. Both IoT service providers and users must give paramount importance for safety. IoT system should ensure the safety both our personal data and the personnel safety. By providing endpoints security, creating a security paradigm is possible.

- Intelligence: IoT system of modern day should make intelligent and smart decisions. In this context Intelligence is a vital characteristic of IoT. Almost every IoT use cases in current world needs Intelligence. The data plays vital role in procuring important business insights and business decisions. Developing Machine Learning or Deep Learning models becomes feasible with the help of massive data. Thus valuable insights can be drawn. Moreover, preprocessing of various analog signals is achieved and is converted to a format that is suitable for training the machine-learning models. Importantly ensuring proper data infrastructure as per the needs of business is crucial.

- Connectivity: As the connectivity ensures achieving better networks that are accessible and compatible. Connectivity is the next important characteristic of a good IoT system. While accessibility provides reachable network the compatibility ensures provides the ability to consume and produce data.

\section{Issues and Challenges for IoT Routing}

Challenging factors that determine the success of routing protocols in IoT networks are summarized in the following section [21]:

- Context Awareness: All the devices of an IoT network can be treated as actors. To ensure fast routing the context information has to be gathered from the environment. Subsequently it is examined for knowledge generation. Thus procured knowledge is used to make routing decisions.

- Heterogeneity: The heterogeneity of components and the corresponding network standards involves handling the intricacy of the routing process. Currently existing protocols operate within rigid boundaries. Naturally, it becomes mandatory to ensure different types heterogeneity unit for all routing protocols.

- Death of nodes: In an IoT network the nodes frequently die due to over usage. As discussed already these nodes are energy constrained. Also exchanging the batteries of nodes frequently is a complex process. Accumulation of dead nodes creates energy holes in the network. This results in short jumps during the routing process due to the short range of operation amongst controlling devices.

- Topology changes: Frequent network topology changes occur in IoT networks due to various factors. Factors like steady mobility of nodes, total energy conservation of nodes and ecological factors primarily bring topology changes. To mitigate such problems developing a hybrid routing protocol or reactive protocol that assists recurrent topology changes is crucial.

- Scalability: Predominantly IoT engross wireless communications. The IoT devices may be both motionless and mobile in nature. Also, the mobile devices entering and leaving the network brings changes in the size of the networks. Obviously this results in the scalability issues.

- Latency: Routing the data generated to the destination node within a stipulated time is the biggest challenge in IoT networks. This is due to the fact that data generated expires within a small span of time so handling Latency is vital in IoT routing services.

- Incentive Based Routing: Cooperation is another important characteristic that governs IoT services. In IoT networks there exists numerous and heterogeneous nodes and ensuring effective communication between all nodes is needed for the successful operation of the network.

- Congestion handling: Congestion in networks is common problem in all types of computer networks. Spike in traffic flow is the main culprit for this intricate mechanism. Congestion mainly leads to problems like packet loss and unwanted delays. Congestion control can be handled using load balancing at every node. Due to congestion the network lifetime is reduced. So, it is important to minimize congestion by using suitable routing protocols.

- Data security features: Obviously the data transmitted in IoT networks have different owners. So it becomes inevitable to ensure security of the data communicated. In addition the entire data in an IoT network is transmitted via wireless mode. So data sleuth is a natural observation. So, to data theft prevention is not easy. Taking measures for Authentication of data transmission between devices becomes inevitable.

- Eliminating data redundancies: IoT networks process a huge quantity of data during the dispensation. Network energy is consumed excessively during such process. Moreover lot of networks energy is wasted processing redundant data. So, data coalition methods are employed to minimize the data redundancy.

- Multipath routing: IoT networks operate efficiently by focusing on two factors load balancing by improving the network lifetime. In addition, Multipath routing facilitates improvement in Reliability, Quality of Services and Fault Tolerance services.

- Limited Resource: Various resource constraints like supply of power, processing power, memory capabilities, bandwidth and range of communication are vital for efficient functioning of IoT devices. Such constraints affect the routing capabilities of IOT networks. To overcome such limitations short distance wireless transmission routing is carried out via multi-hop communication methods. Meaning, data packets are transmitted with the aid of several relay devices until they arrive at the destination. The memory and bandwidth constraint limits the size of the data packet transmitted. Ensuring the IOT devices to transmit the data packets in spite of constrained power source is a challenging task. Also WSN dominates power consumption amongst IOT nodes.

- Node Deployment: Node deployment is a fundamental issue in WSN and IoT networks. This issue can be handled by ensuring node deployment in a proper method in the sensor networks. The node deployment can be done 
automatically (setup) or manually. For manual method, the sensor nodes are arranged using manual methods and the path of data transmission is predetermined. In setup or automatic method the sensor nodes are deployed randomly by employing adhoc routing infrastructure.

- Partitions and Voids: Two other important challenges are Network Partitioning and Voids during routing in IOT networks. Firstly, during partitioning a portion is separated from the network. Further the devices within the partitioned portion are not allowed to communicate with other networks. Secondly void means no any devices exist in the network. Most suitable example for regions of Partitions and Voids is the geographical with lakes and rivers with no WSN communication set up.

IoT Routing Protocols for Energy:
IoT networks functions by continuous interactions between the devices involved in the networks. Various steps involved here are processing, storing and analysis of the information. Care is required to ensure two factors namely: low energy consumption and better scalability. So, any protocols claiming to be efficient must give importance to the aforementioned factors. Routing is the process of transferring data packets from end-to-end which tends to maintain routes prevailing between the wireless network devices. Routing process assigns best possible path or the shortest path to the destination. Also it relies on protocols for data transmission process. Two types of communication methods namely intradomain and interdomain networks can be employed. Broadly identified IoT routing protocols are of three types namely 1 . Network Organization protocols 2. Route Discovery protocols and 3. Protocol Operations. Figure 4.1 depicts routing protocol taxonomy for IoT networks.

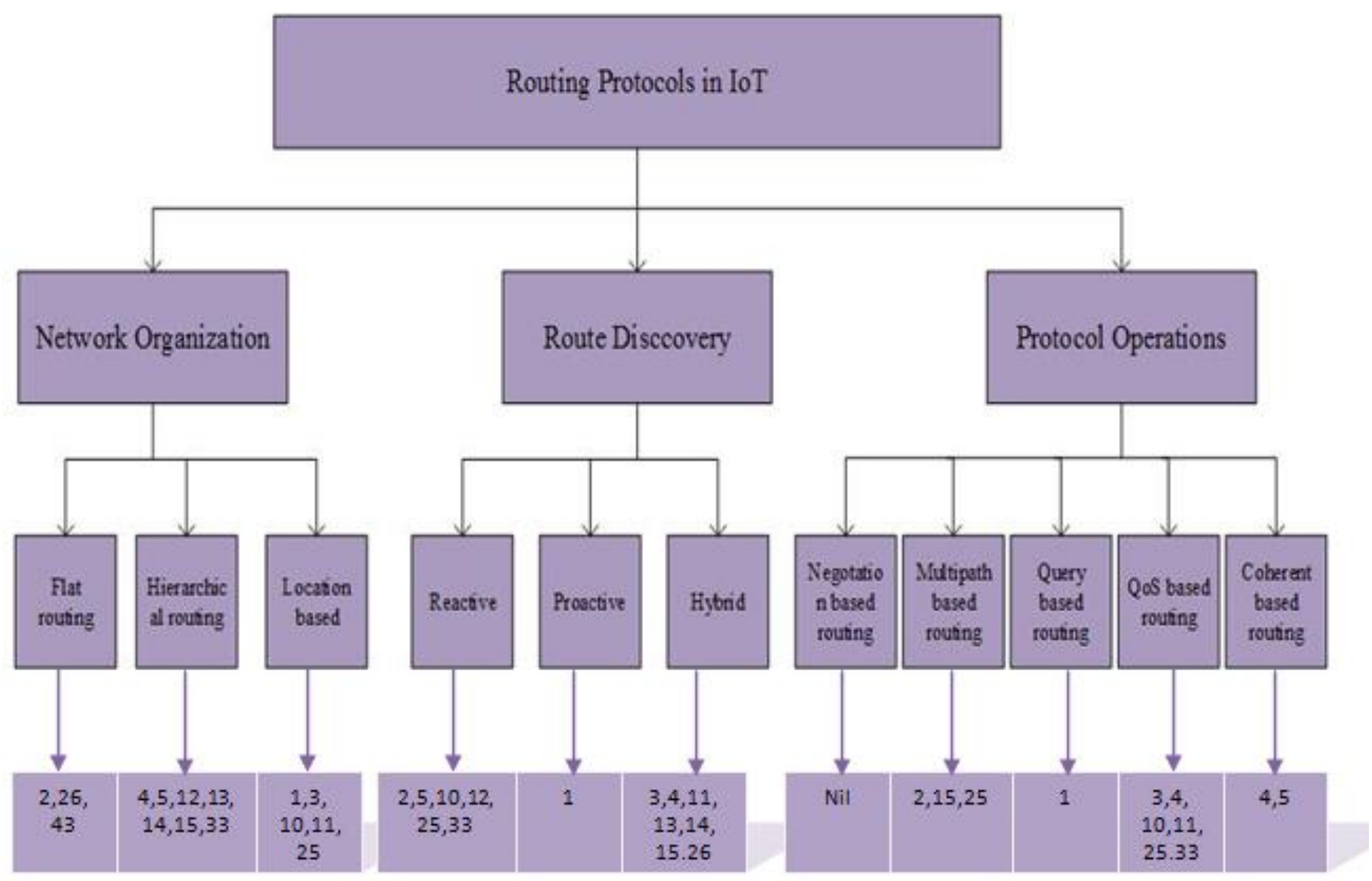

Fig. 4.1 Taxonomy of IoT Energy Routing Protocols

\subsection{Network Organization:}

The network organization is vital in determining routing protocol activities. A brief overview of the network organization features are elucidated in this section. Different protocols for network structure are explained below with corresponding literature review:

4.1.1 Flat-based routing: This routing method is also referred to as horizontal routing. This protocol suits networks having level or horizontal structure. In such networks all nodes are given same priority and they all perform the same role. No special attention or efforts are expected in organizing the networks and their traffic flow. Basically this routing works on contention-based scheduling mechanism. Particularly in IoT networks, it is employed to provide suitable solutions for many independent problems arising because of low operational complexity and high efficiency. This routing is further classified as different types. Examples are DSwEE, EECBR etc.

a) Mohammed Asiri et al [2] have proposed a scheme for improving the data lifespan of IoT devices. In this novel proposal referred as Data Survivability with Energy Efficiency (DSwEE) they have come up with a new-fangled salient simulation metrics called as data recoverable to tests code robustness. This metric recognizes failures or multiple failures happening within a specific network region. DSwEE employs 
a shortest-path routing strategy along with Random Linear Network Coding (RLNC) [43]. The RLNC utilizes coding opportunities for improving data sharing and also save energy during multi-hop forwarding. Initially every node in the network of DSwEE is said to possess the shortest path routing table to destination nodes. This routing applies the identical data survivability model of the original DEC-DS but the dissemination phase differs. First, a source node randomly selects a target storage node in a uniform way. Subsequently, the data source is forwarded by applying the shortest path technique. Unlike DEC-DS, the received data packets are modified by the all relay nodes in the path. This is accomplished by linearly combining them with the previously encoded data packets available on the nodes prior to forwarding. A Redundancy Factor, $m$ is embedded in the packet to track the progress of the code-building process. The factor $\mathrm{m}$ is decremented by one on every local encoding occurring at a newly visited relay node. The process of packet forwarding is terminated with the value of $\mathrm{m}$ becoming 0 . The destination node updates $\mathrm{m}$ if a packet arrives at the destination for a non-zero value of $\mathrm{m}$. Further it randomly selects a new destination storage node and forwards the packet. These steps are repeated in anticipation that $m$ reaches b) Samia Allaoua Chelloug et al [26] have proposed a protocol to diminish the energy consumption in IoT based networks. This protocol is named as Energy Efficient Content-Based Routing (EECBR) protocol. It employs a virtual topology built centrally and the events are routed from the publishers to the subscribers through distributed channel. Also, IoT subscribers and events are matched suitably. Followed by routing of all the events via a virtual topology built from the bottom to the top is achieved. This paper focuses on giving a competent routing service for IoT sensors. These sensors are assigned the role of gathering and routing the data. EECBR differs from conventional routing protocols in two ways: It effectively uses publish or subscribe middleware to accomplish two functionalities: 1) Using virtual topology all IoT sensors are organized. Moreover such virtual topology is formed based on a set of sensors designated for a particular event. In addition the energy residing in the sensors is also taken into account. 2) Using the virtual topology the events are made to hop until they reach the designated sensors. Thus, EECBR is a simple protocol for energy-efficient routing issues of IoT events in a balanced manner. In nutshell EECBR initially creates a virtual topology. Such virtual topology may possess a tree structure or may appear like a set of clusters.

4.1.2 Hierarchical Based Routing: This protocol works by reducing the size of the routing table. Various measures taken in this direction are firstly the network topology is segregated into many layers of hierarchy. By using energy levels as the parameter such layers are created. Such created layers comprises of clusters and cluster heads. In hierarchical method, any node possessing highest energy is designated to send the data and the node having lower energy levels are employed to sense the data. Moreover, Hierarchical algorithms function with Two Layer routing 1) Tree based algorithm and 2) Cluster based Algorithm.

- Tree-based algorithms: This algorithm predicts the traffic pattern by constructing a tree structure relying on communication messages used by IoT devices.
- Cluster-based algorithms: The algorithm splits the sensors into groups using the hierarchical model. Further, the levels in the hierarchical model are employed to determine how the sensor nodes play distinct roles. Any sensor node possessing highest value within a group is voted as a cluster head $(\mathrm{CH})$. The role of $\mathrm{CH}$ is to transmit the information procured from its group to the group members or base station. The main limitation of this algorithm is selecting a cluster head is a time consuming process.

a) Trupti Mayee Behera et al [4] emphasize the need for choosing an efficient cluster head. The process works by regularly updating the cluster head based on the energy levels possessed by a node. In this process the algorithm relies on various parameters to choose a head. The parameters are initial energy, residual energy and an optimum value of cluster heads. Such selection should suit various IoT applications like environmental monitoring and smart cities. The paper highlights how the Low Energy Adaptive Clustering Hierarchy (R-LEACH) protocol can be employed in two phase namely set-up and steady-state phase. During the set-up phase, all the sensor nodes are deployed and are formulated as small groups called clusters. Each cluster will have a designated Cluster Head $(\mathrm{CH})$. The $\mathrm{CH}$ is held responsible for gathering data from the sensing nodes. Redundant bits are eliminated to reduce the volume and the data is fused. In steady-state stage the actual data routing take place. During this phase the $\mathrm{CH}$ forwards the gathered data to the Base Station of the network. b) Khalid Haseeb et al [5] the authors have given an energyaware and secure multi-hop routing (ESMR) protocol. This protocol works on the principle of using a secret sharing scheme to enhance the energy efficiency via multi-hop data security mechanisms to counter malicious actions. The protocol works in three facets: Firstly, based on the location of node segmentation of the network field is accomplished. The identified segments are named as inner and outer zones. Secondly, by using node neighborhood vicinity mechanism numerous clusters are created in each zone. Thirdly, a secure data transmission process is ensured by cluster heads of each zone to the sink node through an efficient secret sharing scheme. Authors have employed ESMR protocol to secret sharing based energy-aware and secure multi-hop routing for IoT based WSNs. The protocol focuses to accomplish routing that is more reliable and energy-aware and avoids packet forwarding by malicious nodes. Using node locations this protocol generates inner and outer zones. Furthermore, Clusters are created by employing the nearest neighborhood scheme, Moreover, a XOR cryptography technique that is lightweight for securing data forwarding in a multi-hop manner is provided for resource constrained networks. Also, this protocol allows the forwarders to communicate data via shortest available route that possesses reliable nodes and energy efficient nodes. Also, authors have given provision to do quantitative analysis of a particular link and recognize congestion thus minimizing routing disturbance and unnecessary re- transmissions.

c) Sonali Sunil Chaudhariet al [12] has come up with a novel protocol for IoT called Mobility Aware Energy Efficient Routing Protocol (MAEER). This protocol is capable of handling mobility and it can discover nearly optimum routes by minimal consumption of energy. This protocol achieves minimal energy consumption by reducing the count of 
participating nodes in $\mathrm{P} 2 \mathrm{P}$ route discovery. Moreover the protocol provides mobility support and increased packet delivery ratio. This protocol functions similar to ER-RPL and computes coordinates, Euclidean distance. The region of each node is determined through Distributive self regioning algorithm. Mobility feature is ensured by MAEER with the help of various mechanisms like dynamic DIS management, region determination stage dynamic DIS and DIO management, and by updating DODAG.

d) P. Venkata Krishna et al [13] The authors have given a proposal on hierarchical frameworks to establish communication in IoT networks. In this work they have developed an energy model to every node. Further by utilizing services associated roles are designated to every node. They have proposed an algorithm for election of optimal cluster head from the pool of available nodes. This is called as Cluster head selection for energy optimization (CHSEO) algorithm. Also, they have proposed a mechanism to minimize the energy loss during communication. Most importantly the framework proposed is successful in efficient placement of sensor nodes. The work culminates with the proposal for CHSEO algorithm for cluster head selection. It works as a solution for energy consumption issues in IoT.

e) T M Behera et al [14] proposed a novel technique based on Dijkstra Algorithm and vector quantization for cluster and active cluster head forming called DEEC-VD. This technique ensures high energy utilization in IoT networks. This technique enables the IoT network to be energy efficient by establishing intra-cluster communication between the cluster heads. It also employs Dijkstra's Algorithm to determine the minimum distance existing between active cluster heads. The proposed algorithm can compute the minimum distance between the active cluster-heads and it aptly suits IoT applications. On considering various metrics it can be inferred that Energy utilization has huge impact on the performance of IoT networks. In addition finding an optimum path to the BS comes in handy for conserving energy.

f) JianShen et al [15] the researchers have come up with a protocol christened as new energy-efficient centroid based routing protocol (EECRP). It is developed for WSN-assisted IoT for improving the network performance. The proposed protocol comprises of three key components: 1) a distributed cluster creation technique for self-organizing local nodes, 2) a novel sequence of algorithms to assure cluster adaptability and cluster head based rotation based on the centroid position for even distribution of the energy load at all sensor nodes, and 3) a novel energy consumption reduction mechanism to handle long-distance communications. EECRP utilizes the residual energy present in the nodes to calculate the centroid position. Authors also have developed an optimization algorithm by employing dead nodes and cluster head nodes count. The simulation results depicts that the EECRP is capable of transmitting a significant quantity of data by minimal energy dissipation provided BS exists within the network.

g) Sang Hyun Park et al [33] the authors have come up with an energy-efficient probabilistic routing (EEPR) algorithm. The algorithm relies on stochastic steps to control the transmission of the routing request packets so that the network lifetime is enhanced and the packet loss is decreased with the flooding algorithm. The proposed EEPR algorithm ensures energyefficiency by simultaneous usage of the residual energy of each node. Also, ETX metric is employed for typical AODV protocol. In addition, for AODV protocol the request packet forwarding is controlled to ensure minimal packet loss and reduced network congestion. The RREQ packet is forwarded to every one-hop neighbor nodes by the source node possessing the data packets. In nutshell, the EEPR algorithm uses residual energy and ETX value for stochastically managing the number of the RREQ packets. Most importantly EEPR algorithm exhibits increased network lifetime and optimally utilizes residual energy of each node in an even fashion as against the typical AODV protocol.

4.1.3 Location Based Routing: This routing technique utilizes signal strength of nodes to identify the location of a node. The information communicated between the nodes is used to notify the relative coordinates and distance between nodes. The transmission route is established by employing region and position of the neighbor nodes. Different types are available namely: E-GIF, SAEER, MEGA, DCEER, NLEE, GAF and GEAR.

a) Ahmed Aboud et al [1] Authors have proposed a power conserving extension to the existing Geographic Interest Forwarding (GIF) protocol. In this proposed E-GIF system they have employed across-layer approach. It let direct communication between protocols at nonadjacent layers. To be precise, MAC and the Routing layer interactions are completely browbeaten to integrate the sleep mode for taking ahead moving decision through the sensor nodes. In this process NDN protocol stack is utilized. The main objective of E-GIF is to reduce the energy consumption and for improvising the network lifetime by regularly turning the radio interfaces $\mathrm{ON} / \mathrm{OFF}$ for the nodes that are not present in the routing path. To accomplish this, the researchers have used duty cycling techniques. During non occurrence of communication the radio transceiver is put in sleep-mode for effective-energy conservation. Ideally, the radio is switched off on non-availability of data and its operation is resumed as soon as new data packet arrives. Thus, nodes alternatively switch over from sleep mode to active mode and viceversa based on network activity.

b) Pallavi Thakur et al [3] SAEER or spectrum aware energyefficient routing protocol is proposed by these authors. In this research work an advanced artificial intelligence methodology-based spectrum aware energy-efficient routing mechanism is employed for D-2-D IoT communication. Authors have used a hybridization of Genetic Algorithm (GA) and Artificial Neural Network (ANN) for providing a secure routing to IoT networks with novelty fitness function. For various routing protocols the Quality of Service is studied using existing and proposed systems and the outcomes are used to validate the optimization by ANN. The focal point of this work is to provide effective district-based information spread issues and to propose a novel SAEER mechanism through GA and ANN by utilizing a new wellness or objective function.

c) Aiswarya S Hampiholi et al [10] have proposed a modified version of GA named as MEGA (Maximum Enhanced Genetic Algorithm). It employs Local Search mechanism along and Sleep-Wake up mechanisms. The Wireless Sensor Networks are optimized so that the energy is conserved and network lifetime is improvised dynamically. The methodology combines the concept of Local Search to implement domain- 
specific knowledge about the networks. Such local search is carried out on each node from each generation of the node population. By effectively applying this mechanism on all nodes energy consumption within the network naturally increases. So, to minimize the energy consumed by the nodes the authors rely on "Sleep-Wake up nodes" mechanism. Also, a sensor in the sleep mode consumes negligible amount of energy and by initiating proper scheduling the right nodes in active state the energy consumption of the networks is well managed and also lifespan enhances.

d) Pinky Bai et al [11] a network layer energy efficient communication protocol for IoT is proposed by these authors. Further the proposed protocol performance is compared with distributed cluster computing energy efficient routing (DCEER). The metrics employed are 1) routing rounds and 2) no of dead nodes in the variable number of nodes rounds. The proposed algorithm functions in two phases: firstly system structure and the secondly basic structure. Getting into system structure it comprises of a Base station and stationary sensing nodes. The sensing nodes are further classified as cluster head $(\mathrm{CH})$ and non-cluster nodes (non - $\mathrm{CH}$ ). In any WSN, a huge chunk of energy is consumed for data communications. The total energy utilized by the network comprises of the energy utilized in transmission of data plus the energy consumed by all sensing nodes.

e) Vellanki $\mathrm{M}$ et al [25] the authors have proposed a node level energy efficient (NLEE) protocol. The objective is to rope in energy efficiency for internet of things. The proposed algorithm has decision making abilities while finding the shortest hop count. The routing metrics employed are transmission count expectations, residual energy available in nodes, and hop count of nodal paths to improvise the energy efficiency. The transmission count expectation can control the requested broadcasts for route discovery. Next, the route discovery process is accomplished via residual energy and hop count of nodal paths. Also, NLEE algorithm ensures improvement in nodal energy usage. During delay in setup or congestion it can provide the shortest path in the network.

4.2 Route Discovery: Route discovery means tracking the routing information from source to destination. It operates based on both reactive and proactive protocols, also it functions employing hybrid protocols.

4.2.1 Reactive protocol: Reactive routing protocol is the one which doesn't rely on node information and the created routes. Usually reactive routing is employed in dynamic networks as they operate with continuously changing topology networks. Prominent Reactive routing protocols are NLEE, MEGA, ESMR, MAEER DSwEE, EEPR etc.

4.2.2 Proactive protocol: This protocol is employed in static networks as the topology is not changed more than once. A tabular format is used to store routing information and this table is referred to as a routing table. Thus, Proactive protocols popularly called as Table- based or Table-driven protocols. Most popular proactive protocol is E-GIF etc.

4.2.3 Hybrid Protocol: They possess properties of both reactive and proactive protocols. Salient Hybrid routing protocols used is: R-LEACH, Zone Routing Protocol, SAEER, DCEER, CHSEO, DEEC-VD, EECRP, EECBR etc.

4.3 Protocol Operation: The main operational characteristics of routing protocols are listed by Protocol operation. Some important characteristics are hierarchy, delivering methods, computation, communication pattern.

a) Multipath Based routing: The salient feature is it possesses ability of undedicated communication for ensuring load balancing and to give better quality of service. Moreover this routing has a fault tolerance mechanism to construct a large number of paths. It also verifies the energy requirements of a single path to communicate periodic messages through such paths. Important challenges faced are obtaining reliability, improve network lifetime and minimize delay. Multipath routing helps in mitigating congestion faced in single path networks. Important Examples are: DSwEE, EECRP, NLEE etc.

b) Negotiation Based Routing: The surplus data present between source and destination nodes is rejected by this Routing mechanism. Also the resource availability is employed negotiating decisions.

c) Query Based Routing: Route discovery is carried in two phases 1) Request phase and 2) Reply phase. During Query phase the data is transmitted between source and destination. The job of query phase is to generate a query packet and transmit the same to nearest nodes. On receiving the node responds to it with a suitable reply. Examples are EGIF etc.,

d) Quality of Service Based Routing: Striking a balance between the energy consumption and data quality is important during QoS-based routing. Most importantly QoS metrics, like delay, energy, bandwidth, etc has to be managed by the networks during data transmission to Base Station. Important QoS Service based routing are: NLEE, RLEACH,MEGA,DCEER, SAEER, EEPR etc.

e) Coherent Based Routing Protocol: Forwarding the data to aggregators with minimal processing is the feature of Coherent Based Routing Protocol. Minimal processing means carrying out tasks like time stamping and suppressing duplicates. By this an energy efficient routing can be achieved. Examples are R-LEACH, ESMR etc.

In Table 4.1 depicts different energy routing protocols that is suitable for distinct classification. Also it contrasts unique steering methods that are in accord with various measurements. 
Table 4.1: Classification and Comparison of IoT Energy Routing Protocols

\begin{tabular}{|c|c|c|c|c|c|c|c|c|c|}
\hline \multirow[b]{2}{*}{$\begin{array}{l}\text { Energy } \\
\text { Routing } \\
\text { Protocols }\end{array}$} & \multicolumn{9}{|c|}{ Various Performance Metrics for IoT } \\
\hline & $\begin{array}{l}\text { Classifi } \\
\text { Cation }\end{array}$ & $\begin{array}{l}\text { Energy } \\
\text { Efficien } \\
\mathrm{t}\end{array}$ & $\begin{array}{l}\text { Dynamic } \\
\text { Topolog } \\
\text { y } \\
\text { Support }\end{array}$ & $\begin{array}{l}\text { Multi } \\
\text { path }\end{array}$ & $\begin{array}{l}\text { Failure } \\
\text { Manage } \\
\text { ment }\end{array}$ & $\begin{array}{l}\text { Link } \\
\text { Quality } \\
\text { Consider } \\
\text { ation }\end{array}$ & $\begin{array}{l}\text { Load } \\
\text { Balancin } \\
\mathrm{g}\end{array}$ & $\begin{array}{l}\text { Multi } \\
\text { hop } \\
\text { Routin } \\
\text { g }\end{array}$ & $\begin{array}{l}\text { Networ } \\
\mathrm{k} \\
\text { Lifetim } \\
\mathrm{e} \\
\text { Improve } \\
\text { ment }\end{array}$ \\
\hline E-GIF [1] & Location & Yes & No & No & Yes & No & No & Yes & Yes \\
\hline DSwEE [2] & Flat & Yes & Yes & Yes & Yes & No & Yes & Yes & Yes \\
\hline SAEER [3] & Location & Yes & Yes & No & Yes & Yes & No & Yes & Yes \\
\hline $\begin{array}{l}\text { RLEACH[4 } \\
\text { ] }\end{array}$ & hierarchical & Yes & Yes & No & Yes & Yes & No & Yes & Yes \\
\hline ESMR [5] & hierarchical & Yes & Yes & No & Yes & No & No & Yes & Yes \\
\hline $\begin{array}{l}\text { MEGA } \\
{[10]}\end{array}$ & Location & Yes & Yes & No & Yes & Yes & No & Yes & Yes \\
\hline $\begin{array}{l}\text { DCEER } \\
{[11]}\end{array}$ & Location & Yes & Yes & No & Yes & Yes & No & Yes & Yes \\
\hline $\begin{array}{l}\text { MAEER } \\
{[12]}\end{array}$ & hierarchical & Yes & Yes & No & Yes & No & No & Yes & Yes \\
\hline $\begin{array}{l}\text { CHSEO } \\
{[13]}\end{array}$ & hierarchical & Yes & Yes & No & Yes & No & No & Yes & Yes \\
\hline $\begin{array}{l}\text { DEEC-VD } \\
{[14]}\end{array}$ & hierarchical & Yes & Yes & No & No & No & No & Yes & Yes \\
\hline $\begin{array}{l}\text { EECRP } \\
{[15]}\end{array}$ & hierarchical & Yes & Yes & Yes & Yes & No & Yes & Yes & Yes \\
\hline NLEE [25] & Location & Yes & Yes & Yes & Yes & Yes & Yes & Yes & Yes \\
\hline $\begin{array}{l}\text { EECBR } \\
{[26]}\end{array}$ & Flat & Yes & Yes & No & Yes & No & No & Yes & Yes \\
\hline EEPR[33] & hierarchical & Yes & Yes & No & Yes & Yes & No & Yes & Yes \\
\hline
\end{tabular}

The Table 1 depicts various performance metrics for IoT development of the routing protocols. Here is a gist of these metrics:

- Energy Efficient: Since saving energy is a crucial parameter in IoT networks the protocols employed should exhibit capabilities to save the energy in the nodes. Obviously, energy efficient routing protocols are desired than those which are energy consuming. Thus, Energy is the primary attribute for choosing from standard routing protocols.

- Dynamic topology support: Dynamicity is important characteristic of IoT networks and using Dynamic topology plays a key role here. Most importantly, this metric should be supported by MANETs.

- Multipath: Reliability in data communication can be realized and Quality of Service (QoS) is improved by using for Multipath Routing techniques. Since Multipath techniques support establishing multiple paths and the best possible path is chosen using salient parameters like the energy prevailing in a node, minimum delay, and minimum hop count this metric plays vital role.

Failure Management: Ensuring minimal node failure and link failures is paramount in IoT networks. So, routing protocols that mitigate such issues are preferred.

- Link Quality: The delay in transmission can be minimized by utilizing good quality path links. Thus, the quality of service in the communication is improved. Always it is better to verify the quality of the path and to maintain and minimize the predicted delays during retransmissions.

- Load Balancing: Multipath metric makes way for a load balanced network. As the chances of failures of nodes are less such networks are highly desirable. Thus, they provide reliable communication.

- Multi-hop Routing: The source to the destination communication involves travelling from various nodes. Such type of routing is called multi-hop routing and it is an important metric.

- Improving the Network Lifetime: The life time of a network is improved by utilizing multipath parameters and the load balancing techniques. As the nodes are resource constrained they operate with limited energy and constant efforts are required to reserve the resources and balance or enhance the lifetime of networks.

\section{Open Research Challenges}

Importantly unique challenges in the arena of designing IoT routing protocols are identified. Moreover, the conventional computer network routing protocols lack such features. Here is a glimpse of such challenges. 
- Security: The first and foremost challenge while designing IoT networks is security issues as they are more vulnerable to attacks. Factors like minimal capacity devices, completely wireless-communication, random failures (both permanent or transient) are the main vulnerability issues any attackers can easily exploit. As the IoT networks operate with minimal hardware capabilities restoring the network to normalcy after an attack is a hurricane task. The system should be capable enough to detect and diagnose the attack and take counter measures through light weight procedures as it is resource constrained.

- Mobility: IoT network implementation demands for Mobility. Due to the presence of ever changing network size and mobile nodes effective Mobility mechanism is key for the success of IoT networks. Frequent interruptions are commonly encountered when data is transmitted between different gateways. Services like caching and tunneling can make networks better by giving access to temporarily unavailable data.

- Interoperability: Interoperability between sources to destination nodes is a big challenge for IoT networks. Moreover, IoT system operates with huge number of heterogeneous devices in wide range of platforms. Handling such heterogeneity networks demands efficient interoperability systems.

- $\quad$ Energy: Energy efficient nodes play vital role in success of an IoT network. So, introducing Energy awareness amongst the routing protocols helps to enhance the life span of a communication link. One proven method of managing energy is to utilize the nodes with residual energy so that life time of link prolongs.

\section{Conclusion}

In this review paper, various issues and challenges in routing protocols are highlighted. Further they are categorized based on parameters like structure of networks, route discovery methods and protocol operations methods. The primary objective of all the routing protocols reviewed in our paper is to minimize the power consumption at sensor nodes and to enhance the network lifetime. On contrary, flat based routing are suitable for smaller networks. The hierarchical routing protocols can handle many sensor nodes through efficient communication mechanism. Also the energy routing protocols reviewed exhibit the capabilities to estimate energy consumption through the process of gathering sensor node's location information. We conclude from our literature review that hierarchical based routing is aptly suited for large networks due to better scalability factor. Also from our survey we could identify prominent simulators used in the arena of IoT and WSN are: Matlab, Contiki OS and NS2.

\section{References}

[1] Aboud, Haifa Touati, BrahimHnish, "Power Saving Extension for The NON-Based - GIF Protocol For the Internet of Things," in National Engineering School of SfaxIResCoMath Research Unit, University of Gabes Tunisia. ISBN: 978-1-53867747-6/19, IEEE 2019, pp.525-530.
[2] Mohammed Asiri, TarekSheltami, "A Novel Approach for Efficient Management of Data Lifespan of IOT Devices," IEEE Internet of Things Journal, doi:10.1109/JIOT.2019.2955099, IEEE 2019, pp.1-9.

[3] Pallavi Thakur, Gunjan Thakur, “Ann Based Route Optimization For Device-To-Device IoT Communication Using An Spectrum Aware Energy Efficient Routing Protocol," Published By: Blue Eyes Intelligence Engineering \& Sciences Publication International Journal of Engineering and Advanced Technology (IJEAT)ISSN: 22498958, Volume-8 Issue-6, August 2019, pp.44884498.

[4] Behera, T. M., Mohapatra, S. K., Samal, U. C., Khan, M. S., Daneshmand, M., \&Gandomi, A. H. (2019). Residual Energy Based Cluster-head Selection in WSNs for IoT Application.IEEE Internet of Things Journal, 1-1. doi:10.1109/jiot.2019.2897119.

[5] Haseeb, K., Islam, N., Almogren, A., Din, I. U., Almajed, H. N., \&Guizani, N. (2019). Secret Sharing-based Energy-aware and Multi-hop Routing Protocol for IoT based WSNs. IEEE Access, 1-1. doi:10.1109/access.2019.2922971.

[6] MoumitaGhosh, RamaSushil ,KaushikGhosh "Detecting and Reporting Forest Fire through Deployment of Three Dimensional Multi-sink Wireless Sensor Network", 4th International Conference on Internet of Things: Smart Innovation and Usages (IoT-SIU), 2019 IEEE, DOI10.1109/IoT-SIU.2019.8777485.

[7] KoichiroIshibashi, DuangchakManyvone, MihoItoh, Van-Lan Dao and Van-Phuc Hoang," Beat Sensors for Smart Environment Monitoring Systems", 3rd International Conference on Recent Advances in Signal Processing, Telecommunications \& Computing (SigTelCom), IEEE2019, pp.77-79.

[8] Y. J. Qu*1, X.G. Ming1, S. Q. Qiu1, Z. W Liu1, X.Y. Zhang1, Z.T. Hou2 "A Framework for Smart Manufacturing Systems Based on the Stakeholders Value," 2018 IEEE International Conference on Advanced Manufacturing (IEEE ICAM 2018).

[9] N. EzzEl Din, M.Azab and A. Mokhtar, "Cooperative IoT Network for Environment and Cost Friendly Transportation", 2018 IEEE 9th Annual Information Technology, Electronics and Mobile Communication Conference(IEMCON), Vancouver, BC, 2018, pp. 965-970.

[10] Hampiholi, A. S., \&Vijaya Kumar, B. P. (2018). Efficient routing protocol in IoT using modified 
Genetic algorithm and its comparison with existing protocols.2018 3rd International Conference on Circuits, Control, Communication and Computing (I4C).doi:10.1109/cimca.2018.8739759.

[11] PinkyBai, Kirshnakumar and Sushilkumar, "Energy Efficient Communication Protocol at Network Layer for Internet of Things" 2018 5thInternational Conference on Signal Processing and Integrated Networks (SPIN) Internet of Things"2018 doi: 9781-5386-3045-7/18/ 2018 IEEE, pp.148-153.

[12] Chaudhari, S. S., Maurya, S., \& Jain, V. K. (2017). MAEER: Mobility aware energy efficient routing protocol for Internet of Things. 2017 Conference on Information and Communication Technology (CICT). doi:10.1109/infocomtech.2017.8340624.

[13] Krishna, P. V., Obaidat, M. S., Nagaraju, D., \&Saritha, V. (2017). CHSEO: An Energy Optimization Approach for Communication in the Internet of Things. GLOBECOM 2017 - 2017 IEEE Global Communications Conference. doi:10.1109/glocom.2017.8255024.

[14] Behera, T. M., Mohapatra, S. K., Mukjerjee, P., \&Sahoo, H. K. (2017). Work-In-Progress: DEECVD: A Hybrid Energy Utilization Cluster-Based Routing Protocol for WSN for Application in IoT. 2017 International Conference on Information Technology (ICIT). doi:10.1109/icit.2017.42.

[15] Shen, J., Wang, A., Wang, C., Hung, P. C. K., \& Lai, C.-F. (2017). An Efficient Centroid-Based Routing Protocol for Energy Management in WSNAssisted IoT. IEEE Access, 5, 18469-18479. doi:10.1109/access.2017.2749606.

[16] A. Gharaibeh, M. A. Salahuddin, S. J. Hussini, A. Khreishah, I. Khalil, M. Guizani, A. Al-Fuqaha, "Smart cities: A survey on data management security and enabling technologies", IEEE Commun. Surveys Tuts. vol. 19, no. 4, pp. 2456 2501, 4th Quart. 2017.

[17] kauevinicius de oliveira, M.Sc.Henri M. EsgathaCastelli, M.sc Sidney josemontebeller, M.Sc Thais G.PradoAvancini "Wireless Sensor Network for Smart Agriculture using ZigBee Protocol”, 2017 IEEE First Summer School on Smart Cities Natal, Brazil, August 6-11, 2017.

[18] Tai-Yeon Ku, Wan-Ki Park, Hoon Choi, "IoT Energy Management Platform for MicroGrid", 7th International Conference on Power and Energy Systems, IEEE2017, pp.106- 110.

[19] S.Y. Mumtaj and A. Umamakeswari, "Neuro fuzzy based healthcare system using IoT", 2017 International Conference on Energy, Communication, Data Analytics and Soft
Computing (ICECDS), Chennai, 2017, pp. 22992303.

[20] S. Kallam, R. B. Madda, C.-Y. Chen, R. Patan, and D. Cheelu, "Low energy-aware communication process in IoT using the green computing approach," IET Networks, vol. 7, no. 4, pp. 258264, 2017.

[21] A Dhumane, R Prasad, J Prasad. Routing Issues in Internet ofThings: A Survey.InProceedings of the InternationalMultiConference of Engineers and Computer Scientists.2016; Vol. 1, 16-1 8.

[22] Keyur K Patel1, Sunil M Patel Internet of ThingsIOT: Definition, Characteristics, Architecture, Enabling Technologies, Application \& Future Challenges, International Journal of Engineering Science and Computing, May 2016.

[23] Z. Xu, L. Chen, C. Chen, and X. Guan, "Joint Clustering and Routing Design for Reliable and Efficient Data Collection in Large-Scale Wireless Sensor Networks," IEEE Internet Things J., vol. 3, no. 4, pp. 520-532, Aug. 2016.

[24] Zigbee,http://www.nxtbook.com/nxtbooks/webcom/ zigbee_rg2016/\#/0, “Zigbee resource guide”, 2016.

[25] M, V., \& SPR, K. (2015). Node Level Energy Efficiency Protocol for Internet of Things.Journal of Theoretical and Computational Science, 03(01). doi:10.4172/2376-130x.1000140.

[26] Chelloug, S.A. (2015) Energy-Efficient ContentBased Routing in Internet of Things. Journal of Computer and Communications, 3, 9-20. http://dx.doi.org/10.4236/jcc.2015.312002.

[27] A. Al-Fuqaha, M. Guizani, M. Mohammadi, M. Aledhari, and M. Ayyash, "Internet of things: A survey on enabling technologies, protocols, and applications," IEEE Communications Surveys \& Tutorials, vol. 17, pp. 2347-2376, 2015.

[28] [28] A. Al-Fuqaha, M. Guizani, M. Mohammadi, M. Aledhari, and M. Ayyash, "Internet of Things: A Survey on Enabling Technologies, Protocols, and Applications," IEEE Commun. Surv. Tutor., vol. 17, no. 4, pp. 2347-2376, 2015.

[29] M. Park, "IEEE 802.11ah: sub-1-ghz licenseexempt operation for the internet of things," IEEE Communications Magazine, vol. 53, no. 9, 2015, pp. 145-151.

[30] M. Nobre, I. Silva, and L. A. Guedes, "Routing and scheduling algorithms for WirelessHART Networks: a survey," in Sensors 15, no. 5, 2015, pp. 9703-9740.

[31] N. Sornin, M. Luis, T. Eirich, T. Kramp, and O.Hersent, "Lorawan specification," LoRa Alliance, January 2015. 
[32] J. Nieminen, T. Savolainen, M. Isomaki, B. Patil, Z. Shelby ,and C. Gomez, "IPv6 over BLUETOOTH(R) Low Energy,” IETF RFC 7668, October 2015.

[33] Park, S.-H., Cho, S., \& Lee, J.-R. (2014). EnergyEfficient Probabilistic Routing Algorithm for Internet of Things. Journal of Applied Mathematics, 2014, 1-7. doi:10.1155/2014/213106.

[34] J. Jin, J. Gubbi, S. Marusic, and M. Palaniswami, "An information framework for creating a smart city through internet of things," IEEE Internet Things J., vol. 1, no. 2, pp. 112-121, 2014.

[35] STAMFORD, “Gartner's 2014 hype cycle for emerging technologies maps the journey to digital business," August 2014, http://www.gartner.com/newsroom/id/2819918.

[36] IEEE802.15.4-2011, "IEEE standard for local and metropolitan area network-part 15.4: Low-rate wireless personal area networks (LR-WPAN)," in IEEE Standards, April, 2012, pp.1-225.

[37] C. Gomez, J. Oller, and J. Paradells, "Overview and evaluation of low-power wireless technology," "iscepin Sensors, vol. 12, no. 9, 2012, pp. 11734-11753.

[38] T. Winter, P. Thubert, A. Brandt, J. Hui, R. Kelsey, P. Levis, K. Pister, R. Struik, J. Vasseur, and R. Alexander, "RPL: IPv6 Routing Protocol for LowPower and Lossy Networks," IETF RFC 6550, March 2012.

[39] Kang Bing, Liu Fu, Yun Zhuo, and Liang Yanlei, "Design of an Internet of Things-based Smart Home System", The 2nd International Conference on Intelligent Control and Information Processing, July 2011, pp. 921-924.

[40] Luigi A., Antonio I., Giacomo M. 2010.The Internet of Things: A survey. Science Direct journal of Computer Networks, Volume 54, Pages: 27872805.

[41] J. Decuir, "Bluetooth 4.0: Low Energy," 2010, https://californiaconsultants.org/wpcontent/uploads/2014/05/CNSV-1205-Decuir.pdf.

[42] A. Kim, F. Hekland, S. Petersen, and P. Doyle, "When hart goes wireless: Understanding and implementing the wirelesshart standard," in IEEE International Conference on Emerging Technologies and Factory Automation, 2008, pp. 899-907.

[43] S.-Y. Li, R. W. Yeung, and N. Cai, "Linear network coding," IEEE transactions on information theory, vol. 49, no. 2, pp. 371-381, 2003. 
